\title{
Ein Briefentwurf von Johannes von Muralt aus dem Jahre 1696
}

\author{
Von Bernhard Peyer, Zürich
}

Es ist mir eine große Freude, zu dem Hans Fischer gewidmeten Festhefte des «Gesnerus» einen kleinen Beitrag beisteuern zu dürfen und damit meine herzlichen Glückwünsche zum Ausdruck zu bringen.

Warum fiel die Wahl ausgerechnet auf ein bloßes Briefkonzept, während noch viele wirkliche Briefe Johannes von Muralts der Veröffentlichung harren? Der Grund liegt darin, daß der Briefentwurf aus einer Zeit stammt, zu der Johannes von Muralt in seinen Briefen vom Latein, das er mühelos beherrschte, zum Gebrauch der lebenden Sprachen überging. In dem Entwurf halten sich Latein und Deutsch die Waage. Zu Anfang führt das Latein; hier hat von Muralt sichtlich vom Latein ins Deutsche übersetzt. Das Ende des Briefentwurfes ist nicht mehr zweisprachlich, sondern deutsch abgefaßt. Leider ist es nicht gelungen, zum Konzept auch den ausgeführten Brief aufzufinden. Deshalb bleibt es ungewiß, in welcher der beiden Sprachen der Brief schließlich geschrieben worden ist.

Im Hinblick darauf, daß nicht alle Leser des «Gesnerus» mit den lokalhistorischen Verhältnissen Zürichs vertraut sein dürften, ist es angezeigt, zur Einleitung einige biographische Angaben über den Schreiber des Briefes sowie über den Adressaten, DAvid SPLEISS in Schaffhausen, und ferner über den im Briefentwurf erwähnten Arzt J. J. WAGNER vorauszuschicken.

Johannes von Muralt, geboren am 18. Februar 1645 in Zürich, studierte in Basel, Leiden, London, Oxford, Paris und Montpellier, promovierte 1671 in Basel und wirkte von da ab bis zu seinem Tode (12. Januar 1733) als Arzt in Zürich. Seit 1688 Stadtarzt, seit 1691 Professor der Physik und Chorherr am Stift zum Großmünster, seit 1721 Archiater. Als Mitglied der Leopoldinischen Akademie führte er den Beinamen Aretäus. Er förderte namentlich die Chirurgie und die Geburtshilfe. Seine zahlreichen Publikationen betreffen die gesamte Medizin sowie verschiedene Gebiete der Naturwissenschaften. Biographische Angaben siehe C. Brunner und W. v. Muralt (1919) sowie B. Peyer (1946).

David Spleiss, geboren am 27.Januar 1659 in Schaff hausen, gestorben ebenda am 11.September 1716. Studierte Medizin und promovierte in Padua. Sohn von Stephan Spleiss, als dessen indirekter Nachfolger er am Collegium humanitatis Professor der Mathematik wurde. In der Folge be- 
kleideten zwei weitere Mitglieder der Familie Splesss die gleiche Stelle. David Spleiss wurde 1711 in Schaffhausen Stadtarzt. Über Fossilien hat er erst 1701 eine kleine Arbeit, den Oedipus osteolithologicus, veröffentlicht, die sich mit den Knochenfunden von Cannstadt befaßt. Von seiner früheren Beschäftigung mit Versteinerungen haben sich keine Zeugnisse erhalten. Biographische Angaben siehe R. Wolf (1858, p. 265-266).

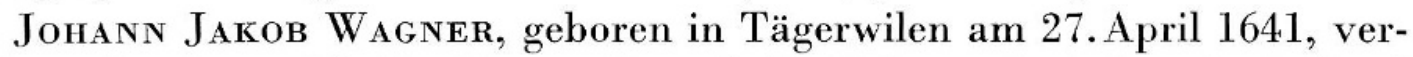
lebte seine Jugend in Zürich, studierte in Heidelberg, Steinfurt, in Holland und in Frankreich Medizin und promovierte in Orange; ließ sich als Arzt in Zürich nieder, wo er 1670 Waisenhausarzt wurde. 1692 wurde er zum 2. Stadtarzt ernannt. Er starb am 14.Dezember 1695. WAGNER war nicht nur ein tüchtiger Arzt, sondern er interessierte sich für die gesamten Naturwissenschaften. Seine Hauptleistung auf diesem Gebiete ist die Historia naturalis Helvetiae curiosa, eine Naturgeschichte des Schweizerlandes. Als Mitglied der Leopoldinischen Akademie führte WaGner den Beinamen Paeon II. Biographische Angaben siehe R. Wolf (1860, p. 93-112) sowie A. Schwarzenberger (1952).

Der Unterschied zwischen lateinischer und deutscher Gelehrtenkorrespondenz zu Ende des 17.Jahrhunderts kommt nicht nur im Briefstil, sondern ebensosehr im Bilde der Handschrift zum Ausdruck. In von Muralts Briefentwurf hat uns die Gunst des Zufalls ein Dokument aufbewahrt, in dem dieser Unterschied uns lebendiger und intimer als in einer sorgfältig ausgeführten Reinschrift in einem und demselben Schriftstück entgegentritt. Um dem Leser auch diese Vergleichsmöglichkeit zu bieten, wurde es daher wünschenswert, das ganze Schreiben bildlich wiederzugeben. Ich bin der Redaktion sehr zu Dank dafür verpflichtet, daß sie diesem Wunsche trotz der großen Raumbeanspruchung entgegenkam. Zu danken habe ich ferner Dr. Rudolf Steiger von der Zentralbibliothek Zürich, dessen Hilfe ich mich bei der genauen Feststellung des Textes erfreuen durfte.

Entwurf eines Briefes von Dr. Johannes von Muralt, Zürich, an Dr. David Spleiss, Schaffhausen, aus dem Jahre 1696. Im Besitz der Zentralbibliothek Zürich, Mscr. H 372, No. 60. Die Jahreszahl 1696 hat von Muralt eigenhändig auf der Rückseite vermerkt. Die deutsch geschriebenen Partien sind kursiv wiedergegeben. 


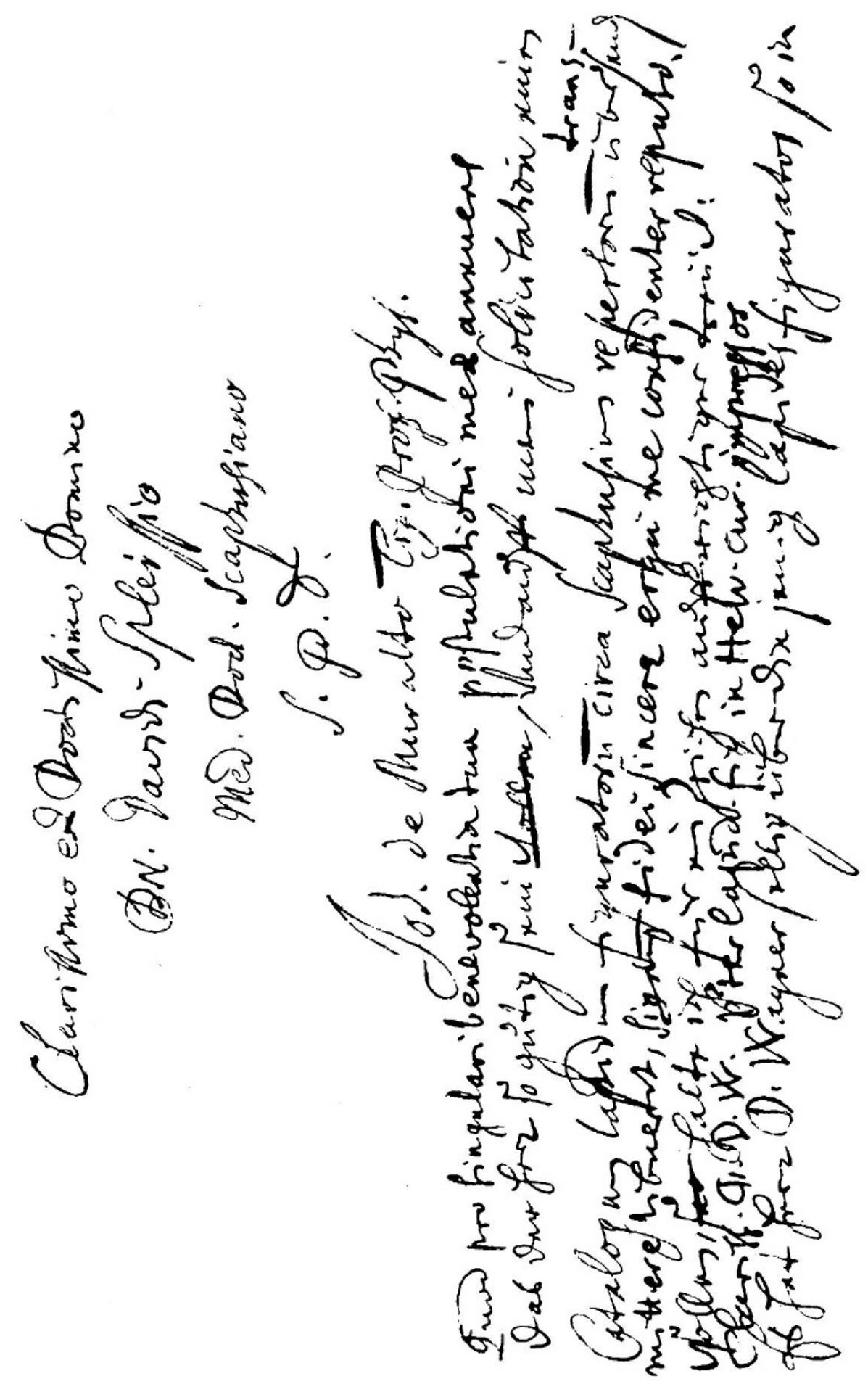




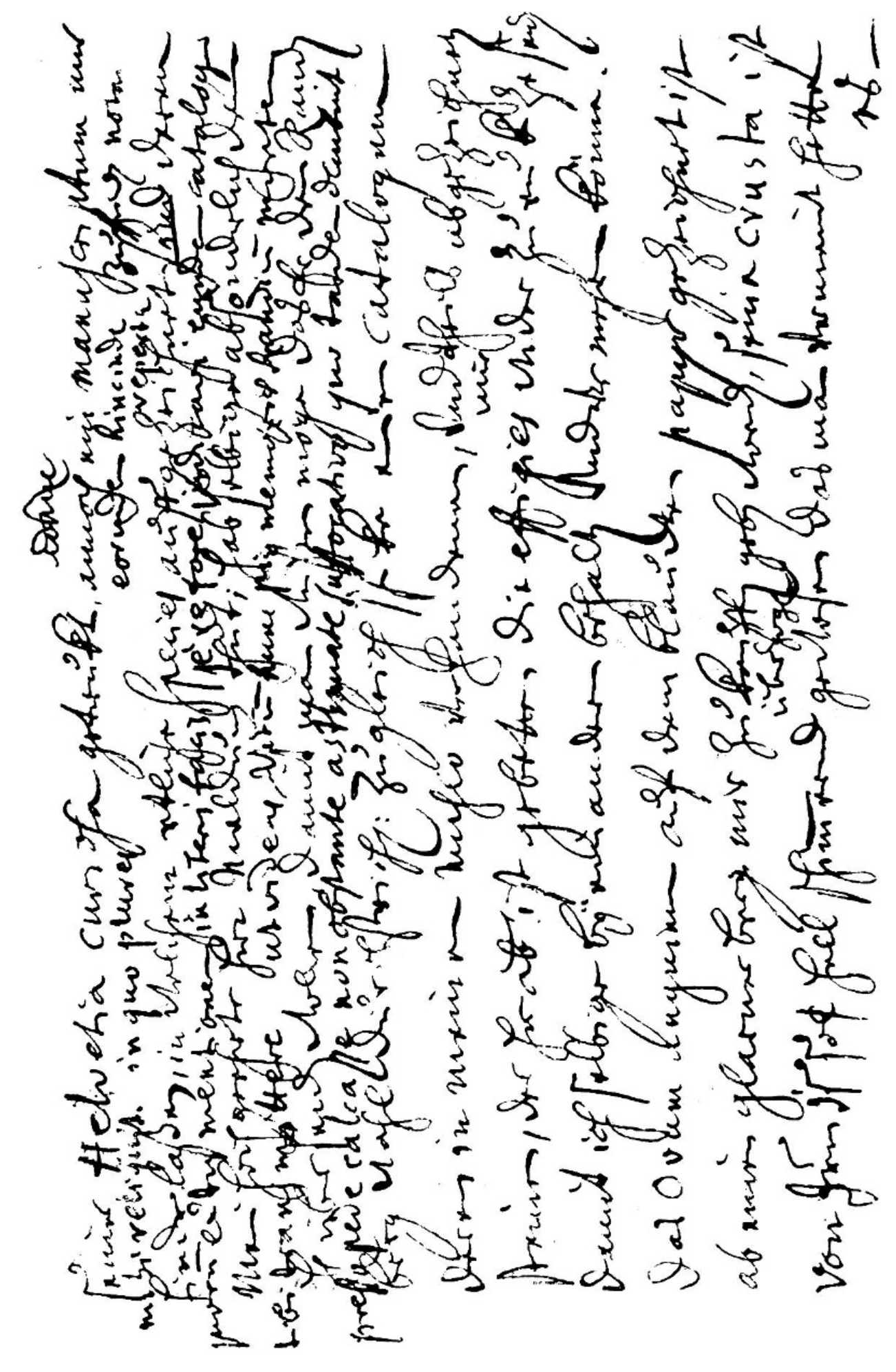




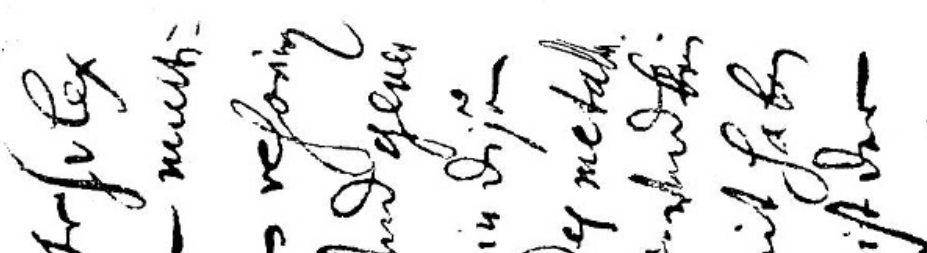
2

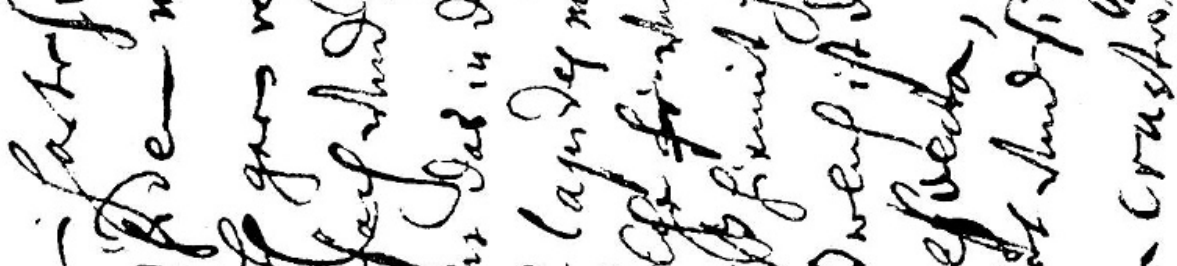

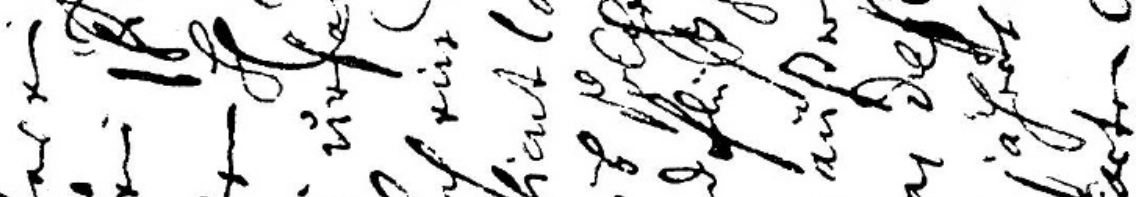

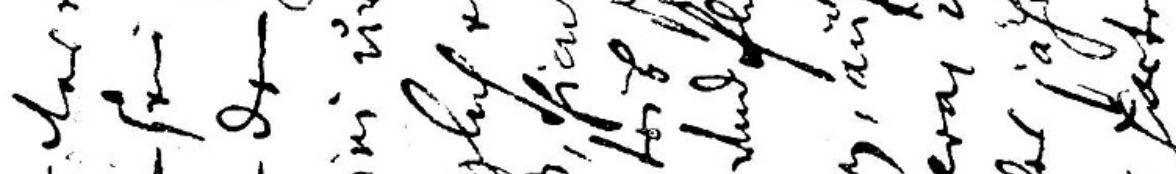

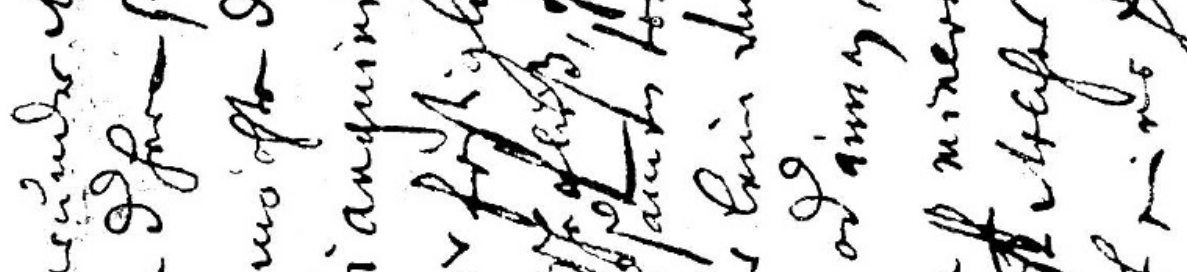

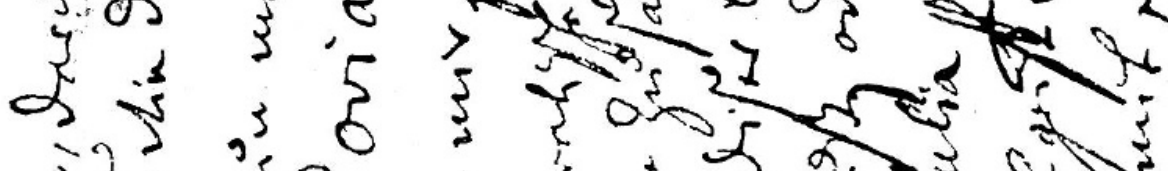

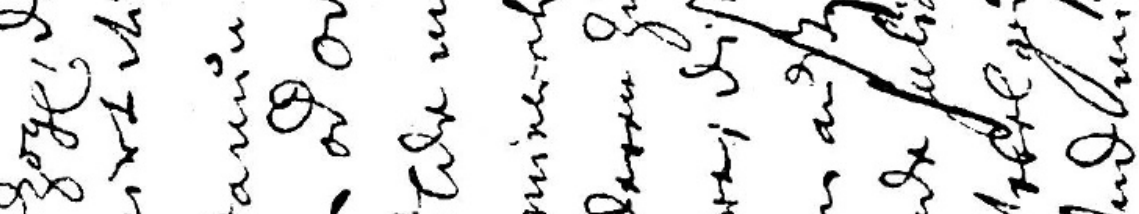

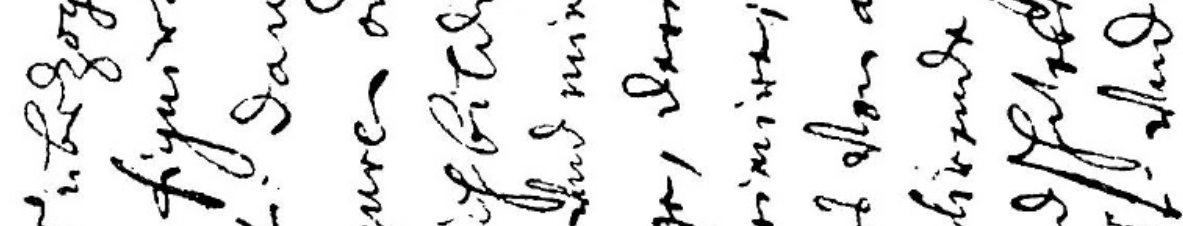

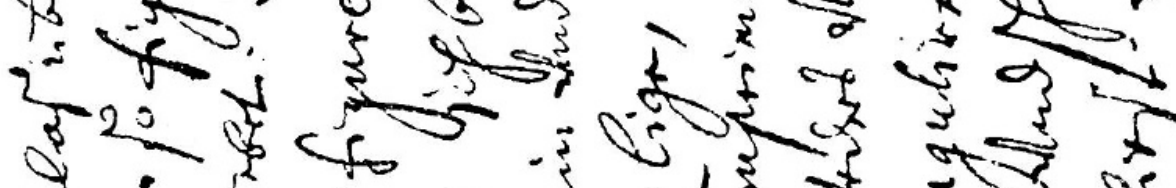

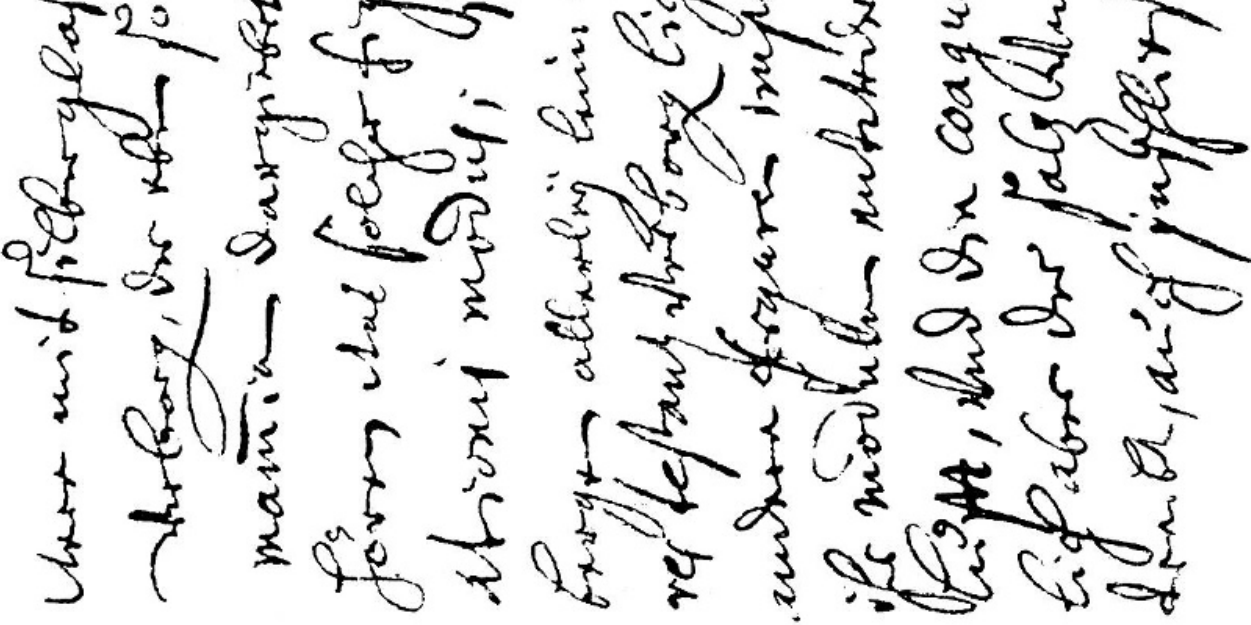




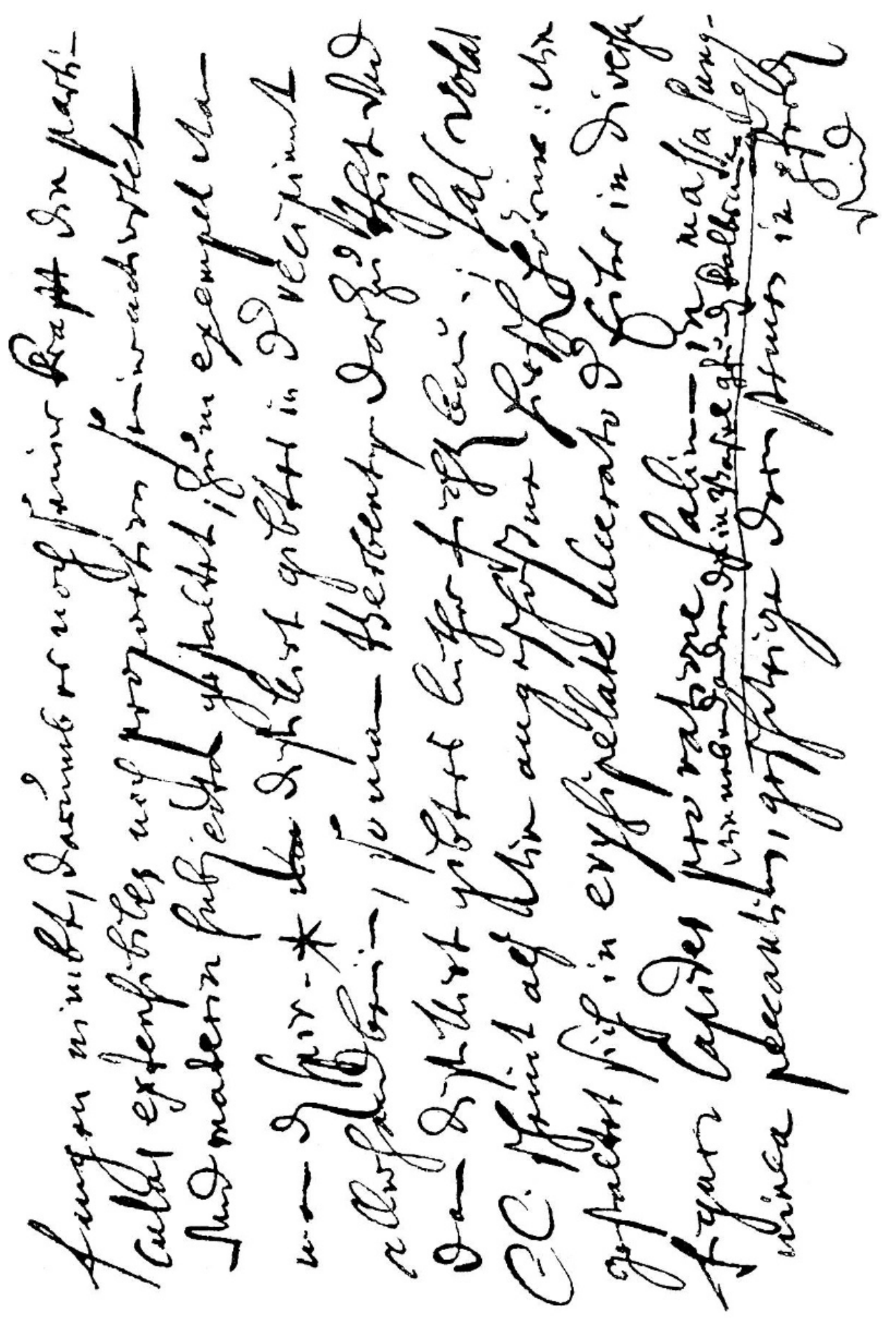




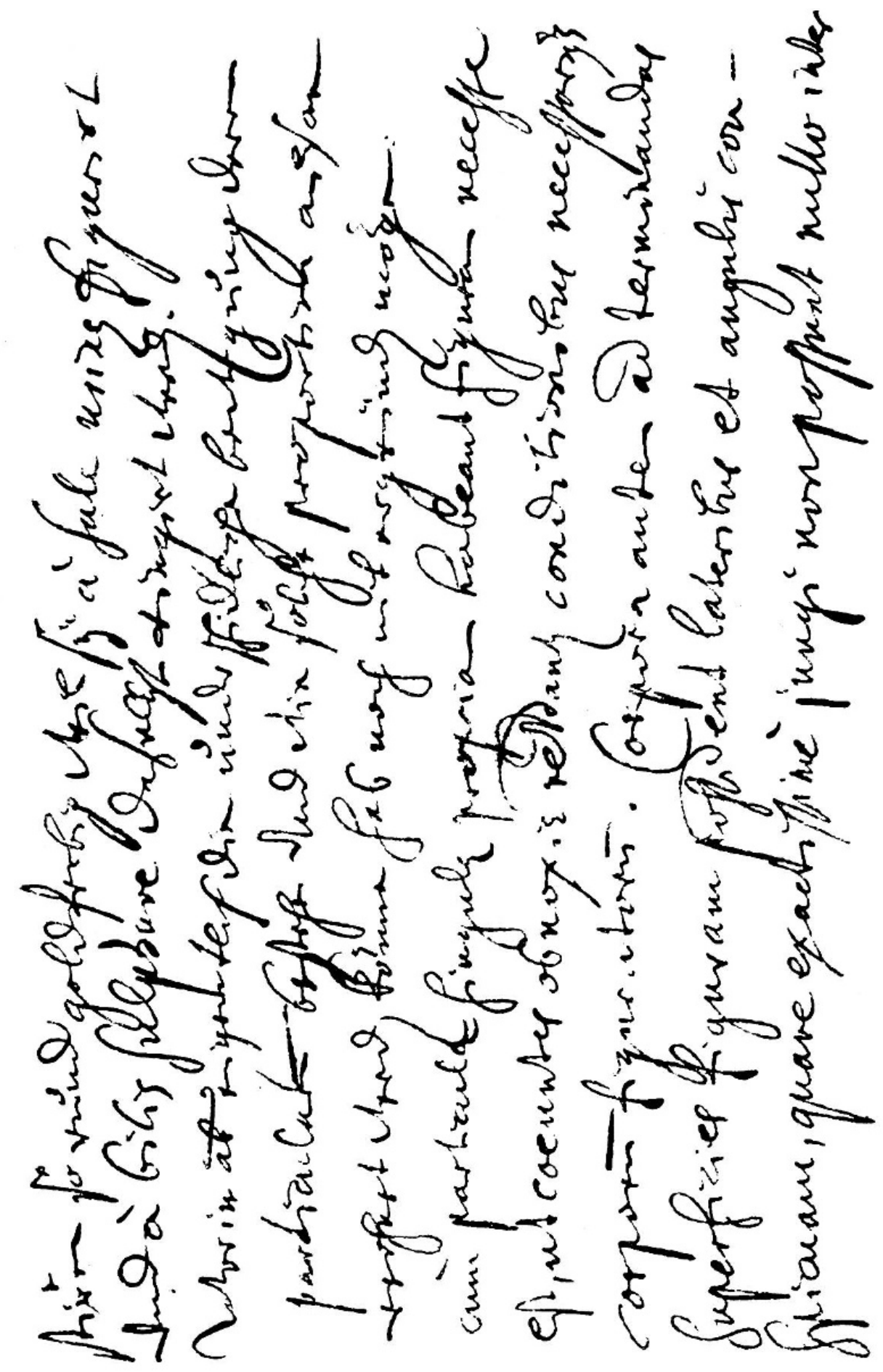




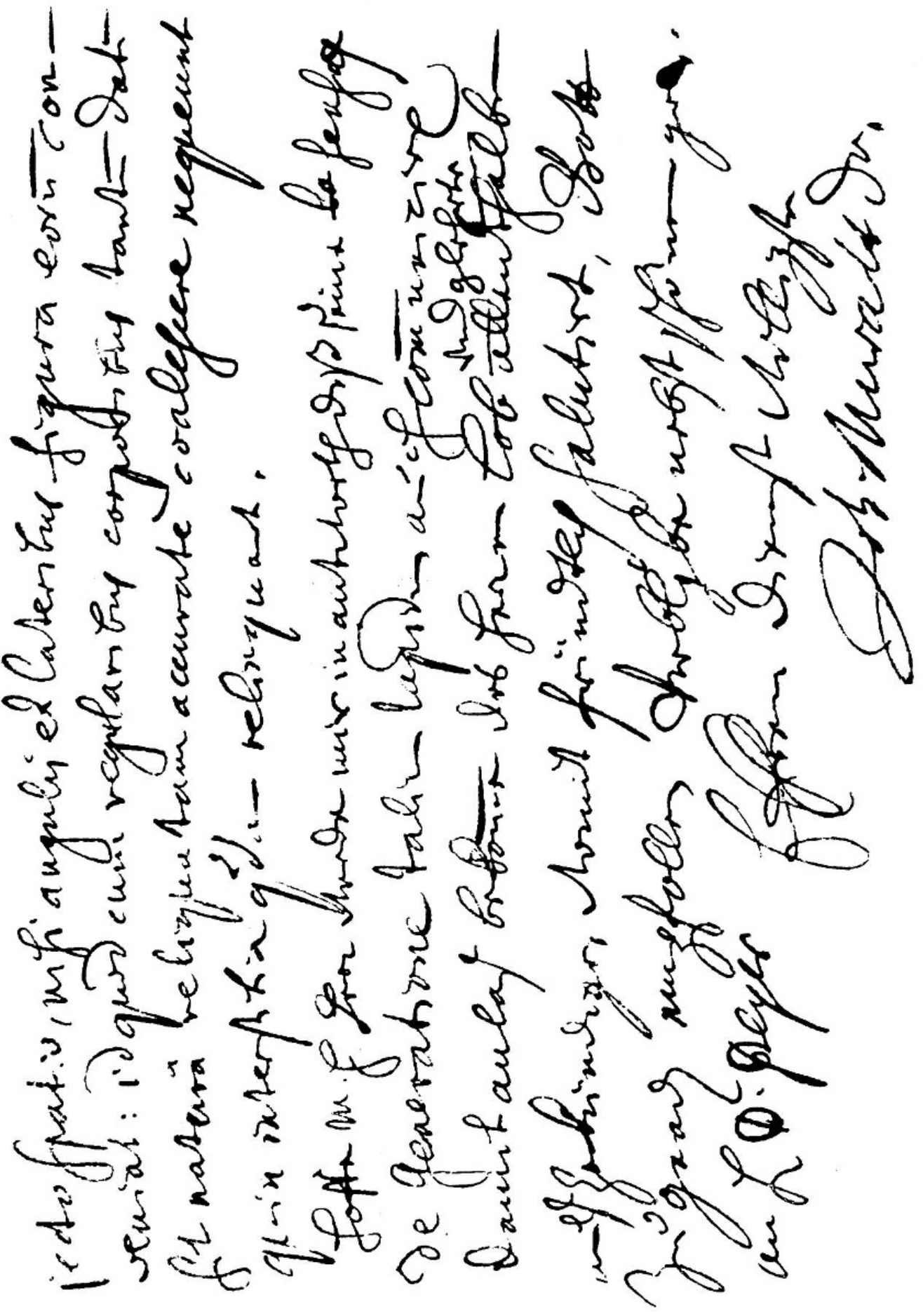


Clarissimo et Doctissimo Domino

DN. Davidi Spleissio

Med. Doct. Scaphusiano

S. P. D.

Joh. de Muralto Tig. Prof. Phys.

Quod pro singulari benevolentia tua postulationi meae annuere das der Herr so gütig sein wollen und auf meine solicitation eines Catalogum lapidum figuratorum circa Scaphusios repertorum transmittere libuerit, signum fidei sincerae erga me confidenter reputo. übersenden wöllen, halte ich für ein Zeichen aufrichtiger treuw. Clariss. D. D. W. praeter lapid. fig. in Helv. cur. impressos Es hat Herr D. Wagner sellig über diejenigen lapides figuratos, so in seiner Helvetia curiosa getrukt, annoch ein Manuskriptum mir mihi reliquit in quo plures eorundum hincinde reperti notantur hinderlassen, in welchem etliche Spezies aufgezeichnet zu finden sind, deren quorum et tu mentionem in literis facis. E re fore judicavi eundem catalogum mein hochgeehrter Herr Meldung thut. Hab selbige absonderlich dem tibi transmittere ut videas virum hunc piae memoriae Randium montem Herrn übersenden wollen, damit man wüssen möge, das Er den Randenpresso pede calcasse non obstante asthmate suffocativo quo tandem decubuit. berg wohl durchstrichen. Zugleich schike einen Catalogum deren in meinem Museo vorhandenen, und theils abgezeichneten Steinen, der Herr aber ist gebetten, die effigies mir wider zurückzesenden damit ich selbige by einanderen behalten und vermehren könne. Das ovum anguinum auf dem blauwen Papyr gezeichnet ist ab einem Glarnerberg mir zu kauffen geben worden, seine crusta ist von Firniß so hell schimmernd überzogen gewesen, das man vermeint hette, es were mit Silberglas überzogen. Darunder war ein harter Silex verborgen, der eben so figuirt, wie der Herr seine Isidem multimammiam dargiebet. Da nun möchte den Herrn gern resoniren hören was solcher Figuren oder ovi anguini ursach und generationis modus; ich bilde mir festiglich ein, das in diesen

Bergen allerley leim und mineralische Flü $\beta$ sicut lapides metalla res testantur verborgen lige, deren Zusammentrib solche und keine andere Figuren imprimire. Diser Leim und Flü $\beta$ hiemit haben ihr modulum entweders von außen oder innen, äußerlich ist der 
Lufft, und die coagulirende salia per mineras devecta, innerlich aber der Salz- und Schwebelgeist welcher jäset und sich treibt, auch jnschließt und mit einer harten crusta sich gefangen nimbt, darumb er nach seiner Kraft die particulas extensibiles nach proportion seiner aktivitet und materia subjecta gestaltet; Zum exempel wan man den Spir.* distillirt gibt es in dem recipient allerhand Bäum, so man Therbentyn darzuthut und dan distilliert, gibt es luter Fichtenbäum; sal. volat. C. C. scheint als wie angeschoßne Hirschhörner: Wie gestaltet sich in erysipelate ulcerato der Eiter in diversas figurae lapides pro ratione salium in massa sanguinea peccantium wie nebend andren die in Basel gefundenen Kalbsaugen geschwige deren Steinen in Pferden und Stiren so rund goldfarbig weil sy a sale urinae figurirt und a bilis sulphure daselbst tingirt werden. Worin aber eigentlich die unterschidliche Bewegung deren particularum bestehe und wie solche proportion außen verhert werden könne, hab noch nit ergründen mögen. cum particulae singulae propriam habeant figuram necesse est, ut coeuntes obnoxie reddantur conditionibus necessariis corporum figuratorum. Corpora autem ad terminandas superficies figuram possident lateribus et angulis conveniat: id quod cum regularibus corporibus tantum datum sit natura reliqua tam accurate coalescere nequeunt quin interstitia quaedam relinquant.

Hoffe M. H. Herr werde mir in antworth diß seine sensus de generatione talium lapidum auch communiciren, damit anla $\beta$ bekomme des Herren lob und glehrte allenthalben aufzekündigen, womit früntlich salutirt, Gott zu gnaden empfollen verblybe nebst schönen grïßen an H. D. Peyer

E. Ehren dienstwilligster Joh. Muralt, Dr. 
Im vorliegenden Zusammenhang interessiert uns vor allem die Form des Schreibens. Hinsichtlich seines sachlichen Inhaltes mögen die folgenden Erläuterungen genügen.

Unter dem Titel De quibusdam lapidibus figuratis Helvetiae hat JoHannes von Muralt eine Anzahl von Fossilien schweizerischer Herkunft beschrieben und auf sieben Tafeln abgebildet. Diese Arbeit erschien 1700 in den Ephemerides der Leopoldinischen Akademie. Im Hinblick auf diese Publikation, deren Manuskript schon im Januar 1697 in die Hände des Herausgebers der Ephemerides gelangte, wandte sich von Muralt an den Schaffhauser David Spleiss in der Hoffnung, von diesem mehr über die auf dem Randen vorkommenden Versteinerungen zu erfahren, als er schon vom Zürcher Waisenhausarzt J. J. WAGNER wußte. WAGNERs 1680 erschienene, lateinisch geschriebene Historia naturalis Helvetiae curiosa, die im Anfang des Briefentwurfes genannt wird, ist die früheste Naturgeschichte des Schweizerlandes. In ihr werden zahlreiche Versteinerungen mit speziellen Fundortsangaben, wie z. B. «in agro Scaphusiano circa Siblingam, Halloviam nec non in monte Randio», «ad radicem Jrchelii montis», «ad locum Eigenthal dictum», «juxta pagum Berg» usw., erwähnt. Wie aus dem Briefentwurf hervorgeht, hat WAGNER das Sammeln von Fossilien auch nach dem Erscheinen seiner Historia naturalis noch fortgesetzt und seinem Kollegen von Muralt Aufzeichnungen darüber hinterlassen.

Das ovum anguinum ist, wie aus den Abbildungen bei Conrad Gessner, Joh. von Muralt und anderen ersichtlich, zweifellos ein fossiler Seeigel; es ist deshalb anzunehmen, daß sich die von D. Spleiss verwendete Bezeichnung Isis multimammia auf die großen Stachelwarzen von Cidaris coronata bezieht.

Im folgenden setzt von Muralt seine Ansichten über die Entstehung der Figurensteine auseinander; er wünscht auch die Meinung von Spleiss hinsichtlich dieser Frage zu erfahren. Eine Antwort von Spleiss hatte ich in der Sammlung der an Joh. von Muralt gerichteten Briefe nicht finden können. Aus einer kleinen Publikation von Spleiss aus dem Jahre 1701, dem Oedipus osteolithologicus (den vollständigen Titel siehe im Literaturverzeichnis) geht jedoch hervor, daß Spleiss sich in dieser Schrift einer bestimmten Meinungsäußerung in der Frage der Entstehung der Versteinerungen enthielt.

Die Diskussion über die Genese der Fossilien dauerte etwa zweihundert Jahre. Moderne Anschaungen hatten schon Leonardo da Vinci, Bernard PALISsy und mehrere italienische Ärzte vertreten. Entscheidend wurde eine 
Arbeit von Nicolaus Steno aus dem Jahre 1669, allein die neue Erkenntnis setzte sich nur langsam durch. Zu den Anhängern der alten Lehre, die in den Fossilien lediglich anorganische Bildungen sah, gehörten auch Schweizer Ärzte aus der Generation von Muralts, wie J. J. Wagner in Zürich und E. Koenig in Basel sowie - etwa bis 1700 - J. J. Scheuchzer. Auch das in den Einzelheiten gute Werk von Karl Nicolaus Lang, Historia lapidum figuratorum Helvetiae, das 1708 erschien, ist noch in diesen Anschauungen befangen. J. J. WAGNER hat 1685 in temperamentvoller Weise gegen Steno polemisiert.

Wie sich Johannes von Muralt die Bildung von Versteinerungen dachte, erfahren wir aus dem Briefentwurf fast anschaulicher als aus der lateinischen Publikation von 1700. Unter Spir.* ist Salmiakgeist zu verstehen, unter Sal. volat. C. C. volatiles Hirschhornsalz. Allen möglichen Steinbildungen im Menschen wie in Tieren wurden im 17.Jahrhundert von ärztlicher Seite häufig Beschreibungen gewidmet.

Als Johannes von Muralt den Entwurf seines Briefes an Spleiss aufsetzte, war er einundfünfzig Jahre alt. Alter Gewohnheit gemäß beginnt er lateinisch, denn in dieser Sprache hatte er seit seinen Jünglingsjahren während über drei Jahrzehnten seine Briefe an Kollegen geschrieben. Nach Überschreitung des fünfzigsten Jahres wechselt man seine Gewohnheiten nur ungern. Was mochte ihn wohl zu der Änderung bewogen haben? Sicher nicht die Person des Adressaten, denn bei dem gelehrten Schaffhauser Arzt durfte eine gründliche Kenntnis des Lateins vorausgesetzt werden. Wir haben uns daran zu erinnern, daß von Muralt zum Entsetzen seiner ärztlichen Kollegen in der Leopoldinischen Akademie schon größere medizinische Werke im Interesse einer Wirkung auf weitere Kreise deutsch geschrieben hatte. Es lag wohl in der Linie dieses Vorgehens, wenn er nun auch in Briefen an deutschsprechende Kollegen zum Gebrauch der deutschen Sprache überging. Als einem weitgereisten Mann ist ihm wohl auch nicht entgangen, daß in England, Frankreich und Italien wissenschaftliche Arbeiten immer mehr in den lebenden Muttersprachen abgefaßt wurden, die damit auch zur Sprache der Akademien wurden. So sind die Mitteilungen in den «Philosophical Transactions» der Royal Society von Anfang an englisch verfaßt. JoH. von Muralt selber war in jungen Jahren in London um eine Mitteilung über die Gletscher der Schweiz gebeten worden. Sein lateinisch verfaßter Aufsatz wurde für die Publikation in den «Transactions» ins Englische übertragen. Nicolaus Steno, der im übrigen lateinisch publizierte, hat doch einen Vortrag, den er in Paris hielt, den 
Discours sur l'anatomie du cerveau, 1669 französisch veröffentlicht. Im Italien des 17. Jahrhunderts finden wir einerseits Gelehrte, die am Gebrauch des Lateinischen festhielten, wie z. B. Marcello Malpighi, und anderseits solche, die das Italienische vorzogen, wie z. B. Francesco Redi. Malpighi lebte von 1628 bis 1694, ReDi von 1626 bis 1697.

Am längsten hielt sich das Latein als Gelehrtensprache im deutschen Sprachgebiete; mußte doch noch mein Großvater seine medizinische Dissertation lateinisch abfassen! Angesichts der Versuche, heute auf künstliche Weise eine allgemein verwendbare Weltsprache zu schaffen, muß man sich fragen, ob der Verzicht auf das Latein, das während des Mittelalters wenigstens für den europäischen Bereich diese Funktion erfüllte, einen Vorteil oder einen Nachteil bedeutete. Die Meinungen sind geteilt. Es kann sich hier nicht darum handeln, das Für und das Wider gegeneinander abzuwägen, sondern nur darum, die Art und Weise des Überganges in dem uns vorliegenden konkreten Beispiel im Einzelnen zu verfolgen.

Zunächst fällt auf, daß sich die deutsche Sprache in ihrem damaligen Zustande nicht gerade zu ihrem Vorteil vom bisher üblichen lateinischen Briefstil unterscheidet. Dies zeigt sich schon in den Eingangs- und in den Schlußformeln. Im Latein zu Anfang das bequeme S.P.D. und am Ende ebenso kurze Schlußwendungen, die aber doch eine feine Nuancierung vom herzlichen Ton bis zur kühlen Höflichkeit gestatteten, im Deutschen ein Schwelgen in Titulaturen und schwülstigen Ergebenheitsbezeugungen. Im Latein das $t u$, im Deutschen die umständliche Anrede in der dritten Person. Das Latein bot eine Fülle von begrifflich scharf umrissenen Fachausdrücken zur Erörterung von medizinischen und von naturwissenschaftlichen Fragen, während es im Deutschen daran vorerst noch fehlte, weil sich in einer lebenden Sprache die Einengung des Gebrauches eines Ausdrucks im Sinne einer scharf definierten Verwendung viel schwerer erreichen läßt als in einer toten Fremdsprache. So sehen wir denn auch, daß von Muralt nicht nur den deutsch geschriebenen Teil des Entwurfes mit lateinischen Worten durchsetzt, sondern gelegentlich ganz ins Latein zurückfällt.

Das Bild der Handschrift verrät den eiligen Entwurf; es ist, als ob die Feder dem Flug der Gedanken kaum zu folgen vermochte. Daher auch die vielen Abkürzungen, bei denen von Muralt im Latein einem bestimmten System folgt, während er im Deutschen selbstverständliche Endungen einfach durch einen im Bogen schräg nach unten verlaufenden Strich andeutet. Die lateinischen Partien sind in einer einfachen, klaren Antiqua geschrie- 
ben; im deutschen Teil des Briefentwurfes sind eine Anzahl von Buchstaben in komplizierter Weise verschnörkelt. Es sieht so aus, als ob die Umständlichkeit der Höflichkeitswendungen des damaligen deutschen Briefstiles sich auch im Schriftbilde widergespiegelt hätte.

Alle diese Unterschiede wären in einer gemächlich ausgeführten Reinschrift jedenfalls noch schärfer hervorgetreten als in einem rasch hingeworfenen Konzept, und doch freuen wir uns darüber, daß sich gerade dieser Briefentwurf erhalten hat, denn er läßt uns den Moment miterleben, in dem ein Gelehrter des 17.Jahrhunderts im Zweifel ist, ob er lateinisch schreiben oder sich der Muttersprache bedienen soll.

Die eine der Sammlungen der an Johannes von Muralt gerichteten Briefe reicht bis zum Jahre 1695, die andere bis zum Jahre 1706. Wahrscheinlich waren auch die später an ihn gerichteten Briefe von ihm selber geordnet und zu Bänden vereinigt worden, allein es besteht kaum eine Hoffnung darauf, daß diese späteren Briefe jemals wieder zum Vorschein kommen werden. Dagegen berechtigt die vollständig erhaltene Korrespondenz Johann Jaков Scheuchzers - er ist 1733, also im gleichen Jahre wie von Muralt, gestorben - zu der Vermutung, da 3 auch im brieflichen Verkehr von Muralts das Lateinische nach 1700 allmählich durch die lebenden Sprachen in den Hintergrund gedrängt wurde.

In dankenswerter Weise stellte mir Dr. R. Steiger folgende Feststellung zur Verfügung: Die im Besitz der Zürcher Zentralbibliothek befindliche Korrespondenz der beiden Brüder Јон. Јаков und Johannes Scheuchzer mit etwa 770 Korrespondenten reicht von 1691 bis 1737. Sie umfaßt 7260 Briefe. Davon sind $52 \%$ lateinisch, 33\% deutsch, $13 \%$ französisch, $1,5 \%$ italienisch geschrieben. Während der ersten Jahrzente der Korrespondenz von Johannes von Muralt dominiert das Latein in viel höherem Maße.

\section{Verzeichnis der zitierten Literatur}

Conrad Brunner und Wilhelm von Muralt (1919), Aus den Briefen hervorragender Schweizer Arzte des 17. Jahrhunderts. Hgg. durch die Stiftung von Schnyder von Wartensee. Basel (B. Schwabe).

Johannes von Muralt (1700), De quibusdam lapidibus figuratis Helvetiae. Observatio XV. Miscellanea curiosa sive Ephemeridum medico-physicarum Germanicarum Academiae Caesareo-Leopoldinae Naturae Curiosorum Decuriae III. Annus quintus et sextus. Anni MDCXCVII \& XCVIII. Frankfurt und Leipzig.

Bernhard Peyer (1946), Die biologischen Arbeiten des Arztes Johannes von Muralt. 1645-1733. Hgg. von der Stiftung von Schnyder von Wartensee, Zürich. Thayngen (Augustin). 
Adolf Schwarzenberger (1952), Der Zürcher Arzt und Naturforscher Johann Jacob Wagner (1641-1695). Vierteljahrsschr. Natf. Ges. in Zürich, Jahrgang 97. Zürich.

David Spleiss (1701), Oedipus osteolithologicus seu dissertatio historico-physica de cornibus et ossibus fossilibus Canstadiensibus. Schaff hausen (Frey).

JohanN Jacob W AGNer (1680), Historia naturalis Helvetiae curiosa. Zürich (H. Lindinner).

- (1685), Lapilli albi Caryophyllos aromaticos referentes. Observatio CLXXXIX. Miscellanea curiosa sive Ephemeridum Academiae naturae curiosorum Decuriae II. Annus tertius. Nürnberg.

RUdolf Wolf (1858-1862). Biographien zur Kulturgeschichte der Schweiz. Zürich (Orell Füßli). 